\title{
An efficient two-step subcellular fractionation method for the enrichment of insulin granules from INS-1 cells
}

\author{
Yan Chen ${ }^{1,2}$, Zhiping Xia ${ }^{1,2}$, Lifen Wang ${ }^{1,2}$, Yong Yu ${ }^{1,2}$, Pingsheng Liu ${ }^{1}$, Eli Song ${ }^{1 \bowtie}$, Tao Xu ${ }^{1 \bowtie}$ \\ 1 National Laboratory of Biomacromolecules, Institute of Biophysics, Chinese Academy of Sciences, Beijing 100101, \\ China \\ 2 University of Chinese Academy of Sciences, Beijing 100049, China
}

Received: 20 January 2015 / Accepted: 3 July 2015 / Published online: 7 August 2015

\begin{abstract}
Insulin is one of the key regulators for blood glucose homeostasis. More than $99 \%$ of insulin is secreted from the pancreatic $\beta$-cells. Within each $\beta$-cell, insulin is packaged and processed in insulin secretary granules (ISGs) before its exocytosis. Insulin secretion is a complicated but well-organized dynamic process that includes the budding of immature ISGs (iISGs) from the trans-Golgi network, iISG maturation, and mature ISG (mISG) fusion with plasma membrane. However, the molecular mechanisms involved in this process are largely unknown. It is therefore crucial to separate and enrich iISGs and mISGs before determining their distinct characteristics and protein contents. Here, we developed an efficient two-step subcellular fractionation method for the enrichment of iISGs and mISGs from INS-1 cells: OptiPrep gradient purification followed by Percoll solution purification. We demonstrated that by using this method, iISGs and mISGs can be successfully distinguished and enriched. This method can be easily adapted to investigate SGs in other cells or tissues, thereby providing a useful tool for elucidating the mechanisms of granule maturation and secretion.
\end{abstract}

Keywords Insulin, Immature insulin secretary granules (iISGs), Mature insulin secretary granules (mISGs), Subcellular fractionation, Density gradient

\section{INTRODUCTION}

Insulin is one of the key regulators for blood glucose homeostasis. Abnormal insulin secretion is considered an essential factor in the progression of diabetes (Weir et al. 2001). More than $99 \%$ of insulin is secreted from the pancreatic $\beta$-cells (Rhodes 2000). Within each $\beta$-cell, insulin is stored in specified organelles known as insulin secretary granules (ISGs), and is released via a regulated secretory pathway. Insulin biogenesis initiates with the synthesis of preproinsulin in the rough endoplasmic reticulum (ER) and the conversion of preproinsulin to

Yan Chen, Zhiping Xia and Lifen Wang have contributed equally to this study.

$\bigotimes$ Correspondence: songali@moon.ibp.ac.cn (E. Song), xutao@ibp.ac.cn (T. Xu) proinsulin. Proinsulin begins to be packaged in the trans-Golgi network (TGN) and is sorted into immature ISGs (iISGs). These iISGs gradually become acidic, and proinsulin undergoes proteolytic cleavage, resulting in the formation of mature bioactive insulin and C-peptide (Docherty and Hutton 1983; Orci et al. 1986; Smeekens et al. 1992; Colomer et al. 1996; Davidson 2004; Steiner et al. 2009). During this maturation process, insulin is crystallized in the form of dense core, and unwanted cargoes and membrane proteins are removed and transported either back to the TGN or endo/lysosome systems (Kim et al. 2006). The newly formed mature ISGs (mISGs) are transported to the plasma membrane to be ready for release in response to calcium stimulation (Borgonovo et al. 2006).

Although SGs have been known for almost 70 years, many questions, including how SG-specific components 
are segregated from molecules destined to different organelles and how SGs mature, remain elusive. Therefore, it is necessary to separate iISGs and mISGs to identify their characteristics and protein contents. An efficient isolation of these two organelles is the first and most important step for the subsequent characterizations. However, because ISG maturation is a highly dynamic process, it is extremely difficult to separate iISGs and mISGs. Several previous studies reported methods to isolate ISGs, but the samples isolated in these works were total ISGs, which contained both iISGs and mISGs (Konrad et al. 1995; Iezzi et al. 1999; Brunner et al. 2007). Another group recently reported the development of a three-step gradient purification procedure combined with Stable Isotope Labeling with Amino acids in Cell culture (SILAC) to further characterize the ISG proteins. However, the fraction purities of iISGs and mISGs were not well verified (Schvartz et al. 2012).

In this study, we developed a modified two-step subcellular fractionation method to simultaneously enrich high-purity iISGs and mISGs from cultured INS-1 cells, a rat-derived $\beta$-cell line. We used a primary discontinuous OptiPrep gradient to isolate two fractions (Fractions 6 and 8) in which insulin was abundant. Immunoblotting results and fraction densities indicated that Fractions 6 and 8 were iISGs and mISGs, respectively. Further enrichment of iISGs and mISGs was accomplished using Percoll solution.

\section{RESULTS}

\section{The two-step enrichment protocol for isolating iISGs and mISGs}

Accurate analyses of ISGs are always based on the quality of iISG and mISG enrichment. For the efficient isolation of iISGs and mISGs, we employed an OptiPrep density gradient to enrich iISGs and mISGs simultaneously. The efficient two-step method was established. Briefly, (1) the cultured cells were homogenized in Buffer A by nitrogen cavitation to obtain whole cells (WC), and after centrifugation, the postnuclear supernatant (PNS) was obtained; (2) the PNS was loaded on top of an initial discontinuous OptiPrep gradient with five concentrations; (3) the fractions were collected and examined by ELISA and Western blot after ultra-centrifugation; (4) the insulin containing fractions were added in certain concentration Percoll buffer followed by ultra-centrifugation; and (5) the fractions containing lower levels of contaminants were collected as iISGs or mISGs.

\section{OptiPrep fractionation of subcellular components}

To isolate iISGs and mISGs, the first step, the enrichment of subcellular fractions, was performed. PNS collected from cultured INS-1 cells was separated on a discontinuous OptiPrep density gradient including five concentrations $(8.8 \%, 13.2 \%, 17.6 \%, 23.4 \%$, and $30 \%)$ (Fig. 1). Twelve fractions were individually collected from the top of the OptiPrep gradient (Fig. 2A). To determine which fractions were the enriched iISGs or mISGs, the insulin content was quantified using an ELISA assay. Insulin concentrations in this experiment were in the range of 5-200 ng of the standard curve for a competitive ELISA (Fig. 2B). Fractions 6 and 8 were observed to exhibit the highest levels of insulin (Fig. 2C). It should be mentioned here that the insulin antibody used in the ELISA assay (Sigma, I2018) has high cross-reactivity with proinsulin. Therefore, both iISGs, which contained a large proportion of proinsulin, and mISGs, which contained a large proportion of insulin, showed high insulin levels. Previous studies had reported that iISGs differ from mISGs in size and density, with mISGs acquiring higher density and larger size after maturation (Tooze et al. 1991; Tooze and Stinchcombe, 1992). In the OptiPrep density gradient, the interface of Fraction 6 was between $13.2 \%$ and 17.6\%, and the interface of Fraction 8 was between $17.6 \%$ and $23.4 \%$, indicating that Fraction 8 was denser than Fraction 6. Therefore, iISGs were considered to be enriched in Fraction 6 and mISGs in Fraction 8.

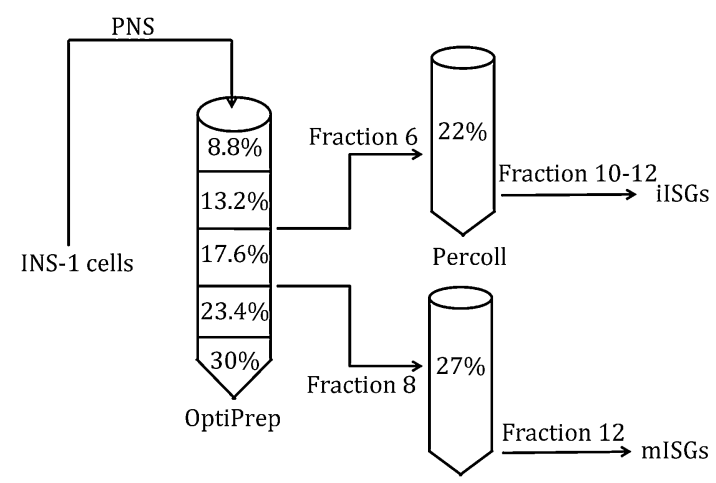

Fig. 1 Schematic illustrating the isolation procedures of iISGs and mISGs from INS-1 cells. Briefly, the INS-1 cell postnuclear supernatant was loaded on top of an OptiPrep gradient consisting of five layers of OptiPrep at varying concentrations. After ultracentrifugation, the two interfaces between $13.2 \%$ and $17.6 \%$ and $17.6 \%$ and $23.2 \%$ were collected as Fractions 6 and 8 . Subsequently, these two fractions were further fractionated using $22 \%$ and $27 \%$ Percoll, respectively. Fractions $10-12$ and Fraction 12 of $22 \% / 27 \%$ Percoll were collected as the final iISG and mISG fractions 
A

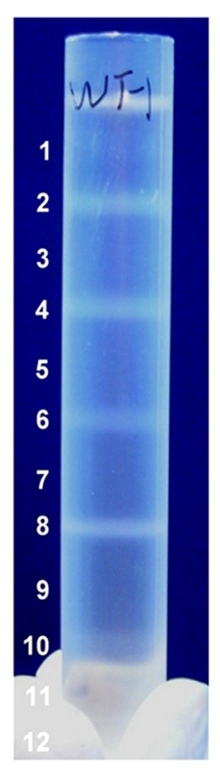

\section{B}

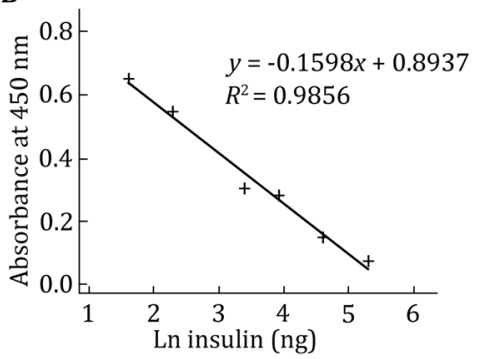

C

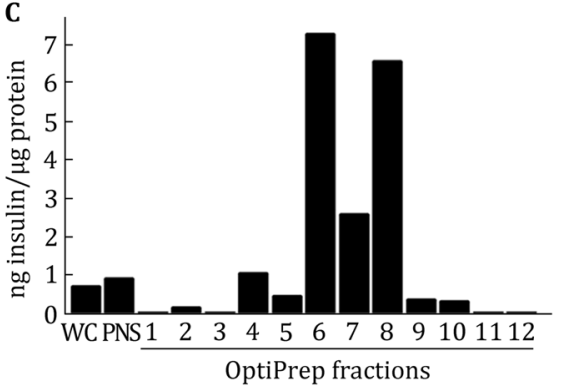

Fig. 2 Analyses of the fractions from the first step of subcellular fractionation. A An example of the fraction distribution after centrifugation. B Standard curve for competitive insulin ELISA. C Insulin quantification in each of the 12 fractions obtained from the OptiPrep gradient

\section{Validation of IISG and mISG fractions}

To characterize each of the 12 fractions isolated from the first step, equal amounts of proteins from each fraction were separated by SDS-PAGE followed by Western blot using antibodies against various markers of cellular compartments, including phogrin, which is a classical ISG-associated membrane protein (Wasmeier and Hutton, 1996); $\beta$-granin, which is a peptide that is co-secreted with insulin and is considered to be a marker for ISG (Hutton et al. 1985); Bip, which is a HSP70 molecular chaperone located in the ER lumen and is hence considered a marker for the ER (Hendershot et al. 1994); TGN46, which is a marker for the TGN

(McCrossan et al. 2001); and GAPDH, which is a marker for the cytoplasm. As shown in Fig. 3A, phogrin and $\beta$ granin were most strongly expressed in Fractions 6 and 8, consistent with the ELISA results, while GAPDH was not detected in Fractions 6 and 8, indicating that these two fractions were not contaminated by cytosolic proteins. However, Bip and TGN46 were also detectable in Fractions 6 and 8, indicating these two fractions were contaminated by ER and Golgi to a small extent.

To further determine iISGs and mISGs, we detected insulin, proinsulin, p18, and Syntaxin-6 in Fractions 6 and 8. p18 is a product of SgII that is processed at several sites by PC2 and accumulates in mISGs; it can be detected using an anti-p18 antibody recognizing only p18 but not larger SgII precursors (Dittie and Tooze 1995; Ahras et al. 2006). Syntaxin-6 functions in the granule maturation process and is localized to TGN and iISG membranes, whereas mISGs have lower levels of this protein (Bock et al. 1996; Klumperman et al. 1998; Wendler et al. 2001). As shown in Fig. 3B, the levels of insulin and p18 in mISGs were higher in Fraction 8 than those in Fraction 6, while less proinsulin and Syntaxin-6 were detected in Fraction 8 than in Fraction 6. These results suggest that iISGs are enriched in Fraction 6 and mISGs in Fraction 8.

\section{Further enrichment of iISGs and mISGs}

Although iISGs and mISGs were successfully enriched in Fractions 6 and 8, each of the fractions had some contaminations. To further enrich iISGs and mISGs, OptiPrep Fractions 6 and 8 were subsequently separated using Percoll solutions. Several concentrations of Percoll, including 20\%,22\%, 25\%, 27\%, and 30\%, were tried first (data not shown), and finally, we determined that the 22\% Percoll solution was suitable for Fraction 6 and the $27 \%$ solution for Fraction 8. Twelve Percoll fractions from Fraction 6 or Fraction 8 were collected. We performed Western blotting to validate each of these
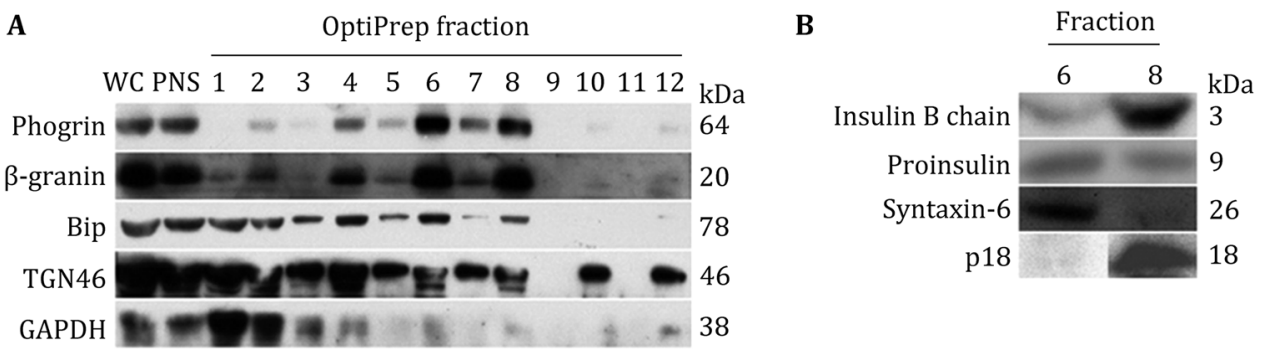

Fig. 3 Western blot assessment of the 12 fractions from OptiPrep gradient. A Western blot analyses of whole cell lysate (WC), postnuclear supernatant (PNS), and the 12 fractions using antibodies against phogrin, $\beta$-granin, Bip, TGN46, and GAPDH. B Western blot analyses of Fraction 6 and Fraction 8 using antibodies against insulin, proinsulin, Syntaxin-6, and p18. Equal amounts of proteins from each fraction were loaded onto SDS-PAGE gels 
fractions using antibodies against three markers including CPE, which is a prohormone-processing enzyme localized to peripheral membrane of ISGs and is used as a marker for ISG; CoxIV, which is a marker for mitochondria (Nakagawa and Miranda 1987); and p62, which is a marker for ER (Mundy and Warren, 1992). As shown in Fig. 4, CPE was successfully enriched, especially in P10, P11, and P12. CoxIV was absent from P6-3 to P6-12, whereas it was observed in all fractions of P8, indicating that this fraction was contaminated by mitochondria. p62 was barely detectable in both Fractions 6 and 8. Western blot analysis showed that in P610 to P6-12, CoxIV and p62 were absent, whereas CPE was present at high levels (Fig. 4A), suggesting that these two fractions were the iISGs fractions. mISGs were well enriched in P8-12 with low levels contaminants. Therefore, we could enrich iISGs in fractions P6-10 to P6-12 and mISGs in fraction P8-12.

\section{DISCUSSION}

Subcellular fractionation has been widely used for the analyses of various intracellular organelles and complexes. In this study, we developed an efficient two-step fractionation strategy for the enrichment of iISGs and mISGs, in which an initial discontinuous OptiPrep gradient was applied, followed by a Percoll solution. This method generated highly enriched fractions of iISGs and mISGs as demonstrated by Western blotting against different intracellular organelle markers.

Our major challenge was the simultaneous isolation of iISGs and mISGs, which are two separate organelles that share many properties. Using a discontinuous

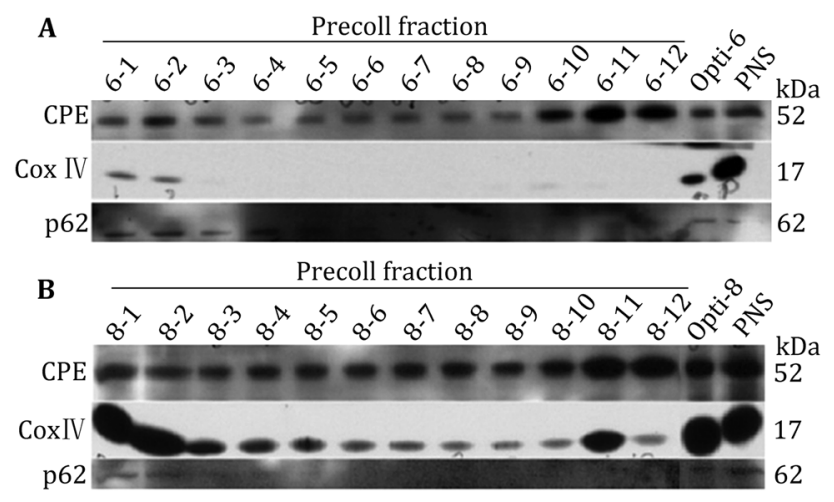

Fig. 4 Western blot analyses of Percoll fractions 1-12 of OptiPrep Fraction 6 (Opti-6) and OptiPrep Fraction 8 (Opti-8) with antibodies directed against CPE, CoxIV, and p62; 0pti-6/8 and PNS were used as controls. A Percoll fractions 1-12 of OptiPrep Fraction 6 (opti-6); B Percoll fractions 1-12 of OptiPrep Fraction 8 (Opti-8). Equal volumes of Percoll fractions were loaded onto SDSPAGE gels
OptiPrep gradient, we successfully separated total ISGs into two fractions, though ER and TGN contaminants were detected in both of them. After the second step, Percoll solution enrichment, we obtained purer iISGs and mISGs fractions, but mitochondria contaminants still existed in the mISG fraction. Generally, complete purification of an organelle by subcellular fractionation is impossible (Huber et al. 2003). There are two main possible reasons for this. First, intracellular organelles are generally highly dynamic entities; this is precisely the case for iISGs and mISGs, the properties and protein contents of which continuously change during the granule maturation process, from budding at the TGN to release at the plasma membrane (D'Amico et al. 2001). This may explain why our enriched fractions were contaminated by TGN contents. Second, a few subcellular structures share similar physical properties in density gradients, which make it difficult to completely separate them. In our experiment, for example, ER contamination was detected in the first step fractionation due to the broad density range of ER-derived membranes (Mast et al. 2010); mitochondria, which are similar to mISGs in density, were still observed in the secondary mISG fraction.

Although the subcellular organelle purification method based on density gradients makes it difficult to avoid contamination by unwanted components, it is still a common and efficient method to enrich our interested organelles. Previously, two proteomic studies were conducted to enrich ISGs followed by proteomic analysis. In the first work, they only enriched total ISGs, without further distinguishing between iISGs and mISGs (Brunner et al. 2007). In the second study, they used a three-step gradient purification procedure to obtain both iISGs and mISGs; however, the properties and purity of the final fractions were not well characterized (Schvartz et al. 2012). Here, we developed a simpler enrichment method and achieved better separation, as verified by many specific markers. Specifically, we used an insulin ELISA and Western blotting against insulin, proinsulin, Syntaxin- 6 , and p18 to determine the iISG and mISG fractions, and we used Western blot against phogrin, $\beta$-granin, Bip, TGN46, GAPDH, CPE, CoxIV, and p62 to determine the purity of our enriched fractions.

ISGs are pivotal organelles of pancreatic $\beta$-cells and represent key participants of glucose homeostasis. It is important to acquire highly purified iISG and mISG fractions for further proteome analyses and biochemical studies to increase our knowledge of ISG biogenesis, maturation, and exocytosis. More complete elucidation of these processes is indispensable for the understanding of $\beta$-cell function and abnormalities in pathologies, such as type 2 diabetes. 
In conclusion, we described a reliable, reproducible, and easy method for the enrichment of mISGs and iISGs from INS-1 cells. This method can be easily adapted to investigate SGs in other cells or tissues.

\section{MATERIALS AND METHODS}

All chemicals were purchased from Sigma-Aldrich with the highest purity available, unless otherwise stated. Percoll solution was obtained from GE Healthcare (17089109). The OptiPrep solution was obtained from Sigma (D1556). A chicken antibody against phogrin was a gift from Dr. Joseph K. Angleson (University of Denver, Denver, USA). The mouse antibody against CPE was from $B D(1: 2000,610758)$. The rabbit antibody against TGN46 was from Sigma (1:1000, T7576). The mouse monoclonal antibody against p18 was from Abcam $(1: 1000, a b 20245)$. The mouse antibody against $\beta$ granin was from Abcam (1:1000, ab81606). The mouse antibody against p62 was from Transduction Laboratories (1:1000, P20120). The mouse antibody against GAPDH was from Chemicon (1:1000, MAB374). The mouse antibody against Bip was from BD (1:1000, 610979). The mouse antibody against Syntaxin- 6 was from Abnova (1:1000, H00010228-M01). The guinea pig antibody against insulin was from Abcam (1:1000, ab7842). The mouse antibody against proinsulin was from HyTest (1:1000, 2PR8). The antibody against CoxIV was a gift from Dr. Pingsheng Liu (Institute of Biophysics, Beijing, China).

\section{Cell culture}

INS-1 cells were cultured in regular RPMI-1640 medium (Gibco) supplemented with $11.1 \mathrm{mmol} / \mathrm{L} \mathrm{D}$-glucose, $10 \%$ fetal bovine serum (Gibco), $1 \mathrm{mmol} / \mathrm{L}$ sodium pyruvate (Sigma), and $50 \mu \mathrm{mol} / \mathrm{L}$ mercaptoethanol (Sigma) and maintained at $37^{\circ} \mathrm{C}$ and $5 \% \mathrm{CO}_{2}$ in a humidified incubator.

\section{Isolation of insulin secretory granules}

iISGs and mISGs were obtained by subcellular fractionation from INS-1 cells. All the following procedures were performed at $4{ }^{\circ} \mathrm{C}$. Briefly, two $150-\mathrm{mm}$ plates of INS-1 cells were washed once with ice-cold PBS and scraped in ice-cold PBS with $1 \mathrm{mmol} / \mathrm{L}$ PMSF. Cells were then homogenized in $5 \mathrm{~mL}$ of Buffer $\mathrm{A}(0.3 \mathrm{~mol} / \mathrm{L}$ sucrose, $1 \mathrm{mmol} / \mathrm{L}$ EDTA, $1 \mathrm{mmol} / \mathrm{L} \mathrm{MgSO}_{4}, 10 \mathrm{mmol} / \mathrm{L}$ MES-KOH, pH 6.5) containing $1 \mathrm{mmol} / \mathrm{L}$ PMSF by nitrogen cavitation for $15 \mathrm{~min}$ at $450 \mathrm{psi}$ on ice. The homogenate (whole cell) was centrifuged at $1000 \mathrm{~g}$ for
5 min to remove unbroken cells and nuclear debris. The supernatant was collected and loaded on top of a discontinuous Optiprep gradient composed of 5 layers

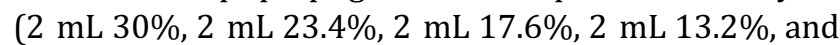
$2 \mathrm{~mL} \mathrm{8.8 \% ).30 \%} \mathrm{OptiPrep} \mathrm{was} \mathrm{prepared} \mathrm{as} \mathrm{stock} \mathrm{in}$ Buffer B $(2 \mathrm{mmol} / \mathrm{L}$ EGTA, $20 \mathrm{mmol} / \mathrm{L}$ MES-KOH, pH $6.5)$, and the rest were made by diluting $30 \%$ OptiPrep with Buffer A containing $1 \mathrm{mmol} / \mathrm{L}$ PMSF in a SW40 tube, and the sample was centrifuged at $100,000 \mathrm{~g}$ for $75 \mathrm{~min}$. On the basis of visible interface, each fraction was collected in appropriate $600 \mu \mathrm{L}$. Fraction 6 (interface between $13.2 \%$ and $17.6 \%$ ) and Fraction 8 (interface between $17.6 \%$ and $23.4 \%$ ), which contained the most insulin, were considered to be the IISG and mISG fractions, respectively. In the second gradient centrifugation, we used Percoll instead of Optiprep (Sigma, D1556). $2 \mathrm{~mL}$ diluted Fraction 6 was mixed with $10 \mathrm{~mL}$ $22 \%$ Percoll solution $(2.2 \mathrm{~mL}$ Percoll diluted to $10 \mathrm{~mL}$ with modified Buffer A containing $0.27 \mathrm{~mol} / \mathrm{L}$ sucrose instead of $0.3 \mathrm{~mol} / \mathrm{L}$ ) and $2 \mathrm{~mL}$ diluted Fraction 8 was loaded on top of $10 \mathrm{~mL} 27 \%$ Percoll solution $(2.7 \mathrm{~mL}$ Percoll diluted to $10 \mathrm{~mL}$ with Buffer A). Then the Percoll samples were centrifuged at $35,000 \mathrm{~g}$ for $45 \mathrm{~min}$. The top $1 \mathrm{~mL}$ was discarded, and the remainder was split in $\sim 0.9 \mathrm{~mL}$ aliquots for the following experiments.

\section{Quantification of insulin levels in isolated insulin secretory granules}

The insulin contents of the ISG fractions were measured using a competitive ELISA assay. Before measurement, $0.05 \mathrm{~g} / \mathrm{mL}$ insulin (Sigma, I0516) was used to coat a Nunc immuno-module 96-well plate overnight at $100 \mu \mathrm{L} /$ well. Stepwise insulin concentrations from 0 to $400 \mathrm{ng}$ were mixed and preincubated with $30 \mathrm{ng}$ of mouse anti-insulin antibody (Sigma, I2018) in $100 \mu \mathrm{L}$ of PBST (PBS with $0.05 \%$ tween-20) in a $1.5-\mathrm{mL}$ tube for $1 \mathrm{~h}$. Meanwhile, the coated plate was blocked by $0.2 \%$ BSA in PBST for $1 \mathrm{~h}$ after washing 3 times with $300 \mu \mathrm{L} /$ well in PBST. The mixture of insulin and the anti-insulin antibody was added in triplicate to the insulin-coated wells, and the plate was incubated for $1 \mathrm{~h}$. After incubation, the plate was washed three times with PBST, $300 \mu \mathrm{L} /$ well. The plate was then incubated with BiotinSP-conjugated goat anti-mouse IgG $(\mathrm{H}+\mathrm{L})$ (Jackson ImmunoResearch, code number: 115-065-003, $1: 30,000$ ) for $1 \mathrm{~h}$, followed by another incubation with peroxidase-conjugated streptavidin (based on the recommended concentration) for $1 \mathrm{~h}$ after washing six times with $300 \mu \mathrm{L} /$ well of PBST. Finally, $50 \mu \mathrm{L}$ of peroxidase substrate (TMB) was added, and the plate incubated for $10 \mathrm{~min}$ followed by addition of $50 \mu \mathrm{L}$ of 
$0.3 \mathrm{~mol} / \mathrm{L} \mathrm{HCl}$ to stop the reaction. Absorbance was measured at $450 \mathrm{~nm}$.

\section{Western blot}

Protein concentration was determined with the bicinchoninic acid assay (Thermo Scientific, 23227). Proteins were separated by SDS-PAGE (insulin and proinsulin were separated in Novex 4-12\% Bis-Tris gel (Life Technologies, 12050371)) and were then transferred to PVDF membranes. The membranes were first blocked for $1 \mathrm{~h}$ with $5 \%$ non-fat dry milk in TBST, incubated for 1-2 h with primary antibody, washed 4 times for $5 \mathrm{~min}$ each time in TBST, incubated for $1 \mathrm{~h}$ with the appropriate peroxidase-conjugated secondary antibody, washed 4 times for 7 min each time in TBST, and then developed photographically by ECL.

Acknowledgments This work was supported by the National Natural Science Foundation of China (31130065, 31270884), the Beijing Natural Science Foundation (5122026) and Youth Innovation Promotion Association CAS.

Compliance with Ethics Guidelines Yan Chen, Zhiping Xia, Lifen Wang, Yong Yu, Pingsheng Liu, Eli Song and Tao Xu declare that they have no conflicts of interest. This article does not contain any studies with human or animal subjects performed by the any of the authors.

Open Access This article is distributed under the terms of the Creative Commons Attribution 4.0 International License (http:// creativecommons.org/licenses/by/4.0/), which permits unrestricted use, distribution, and reproduction in any medium, provided you give appropriate credit to the original author(s) and the source, provide a link to the Creative Commons license, and indicate if changes were made.

\section{References}

Ahras M, Otto GP, Tooze SA (2006) Synaptotagmin IV is necessary for the maturation of secretory granules in PC12 cells. J Cell Biol 173:241-251

Bock JB, Lin RC, Scheller RH (1996) A new syntaxin family member implicated in targeting of intracellular transport vesicles. J Biol Chem 271:17961-17965

Borgonovo B, Ouwendijk J, Solimena M (2006) Biogenesis of secretory granules. Curr Opin Cell Biol 18:365-370

Brunner Y, Coute Y, Iezzi M, Foti M, Fukuda M, Hochstrasser DF, Wollheim CB, Sanchez JC (2007) Proteomics analysis of insulin secretory granules. Mol Cell Proteomics 6:1007-1017

Colomer V, Kicska GA, Rindler MJ (1996) Secretory granule content proteins and the luminal domains of granule membrane proteins aggregate in vitro at mildly acidic $\mathrm{pH}$. J Biol Chem 271:48-55

D’Amico F, Skarmoutsou E, Imbesi RM, Sanfilippo S (2001) Early events of secretory granule formation in the rat parotid acinar cell under the influence of isoproterenol. An ultrastructural and lectin cytochemical study. Eur J Histochem 45:169-175
Davidson HW (2004) (Pro)insulin processing-a historical perspective. Cell Biochem Biophys 35(1):143-157

Dittie AS, Tooze SA (1995) Characterization of the endopeptidase PC2 activity towards secretogranin II in stably transfected PC12 cells. Biochem J 310(Pt 3):777-787

Docherty K, Hutton JC (1983) Carboxypeptidase activity in the insulin secretory granule. FEBS Lett 162:137-141

Hendershot LM, Valentine VA, Lee AS, Morris SW, Shapiro DN (1994) Localization of the gene encoding human Bip/Grp78, the endoplasmic-reticulum cognate of the Hsp70 family, to chromosome-9q34. Genomics 20:281-284

Huber LA, Pfaller K, Vietor I (2003) Organelle proteomicsimplications for subcellular fractionation in proteomics. Circ Res 92:962-968

Hutton JC, Hansen F, Peshavaria M (1985) Beta-granins: $21 \mathrm{kDa}$ co-secreted peptides of the insulin granule closely related to adrenal medullary chromogranin A. FEBS Lett 188:336-340

Iezzi M, Escher G, Meda P, Charollais A, Baldini G, Darchen F, Wollheim CB, Regazzi R (1999) Subcellular distribution and function of Rab3A, B, C, and D isoforms in insulin-secreting cells. Mol Endocrinol 13:202-212

Kim T, Gondre-Lewis MC, Arnaoutova I, Loh YP (2006) Dense-core secretory granule biogenesis. Physiology 21:124-133

Klumperman J, Kuliawat R, Griffith JM, Geuze HJ, Arvan P (1998) Mannose 6-phosphate receptors are sorted from immature secretory granules via adaptor protein AP-1, clathrin, and syntaxin 6-positive vesicles. J Cell Biol 141:359-371

Konrad RJ, Young RA, Record RD, Smith RM, Butkerait P, Manning D, Jarett L, Wolf BA (1995) The heterotrimeric G-Protein $\mathrm{G}(\mathrm{I})$ is localized to the insulin secretory granules of beta-cells and is involved in insulin exocytosis. J Biol Chem 270:12869-12876

Mast S, Peng L, Jordan TW, Flint H, Phillips L, Donaldson L, Strabala TJ, Wagner A (2010) Proteomic analysis of membrane preparations from developing Pinus radiata compression wood. Tree Physiol 30:1456-1468

McCrossan M, Windsor M, Ponnambalam S, Armstrong J, Wileman $\mathrm{T}$ (2001) The trans Golgi network is lost from cells infected with African swine fever virus. J Virol 75:11755-11765

Mundy DI, Warren G (1992) Mitosis and inhibition of intracellular transport stimulate palmitoylation of a $62-\mathrm{kD}$ protein. J Cell Biol 116:135-146

Nakagawa M, Miranda AF (1987) A monoclonal antibody against cytochrome c oxidase distinguishes cardiac and skeletal muscle mitochondria. Exp Cell Res 168:44-52

Orci L, Ravazzola M, Amherdt M, Madsen O, Perrelet A, Vassalli JD, Anderson RGW (1986) Conversion of proinsulin to insulin occurs coordinately with acidification of maturing secretory vesicles. J Cell Biol 103:2273-2281

Rhodes C (2000) Processing of the insulin molecule. In: Diabetes Mellitus: a fundamental and clinical text, p 27-50

Schvartz D, Brunner Y, Coute Y, Foti M, Wollheim CB, Sanchez JC (2012) Improved characterization of the insulin secretory granule proteomes. J Proteomics 75:4620-4631

Smeekens SP, Montag AG, Thomas G, Albigesrizo C, Carroll R, Benig M, Phillips LA, Martin S, Ohagi S, Gardner P et al (1992) Proinsulin processing by the subtilisin-related proprotein convertases furin, Pc2, and Pc3. Proc Natl Acad Sci USA 89:8822-8826

Steiner DF, Park SY, Stoy J, Philipson LH, Bell GI (2009) A brief perspective on insulin production. Diabetes Obes Metab 11:189-196

Tooze SA, Stinchcombe JC (1992) Biogenesis of secretory granules. Semin Cell Biol 3:357-366 
Tooze SA, Flatmark T, Tooze J, Huttner WB (1991) Characterization of the immature secretory granule, an intermediate in granule biogenesis. J Cell Biol 115:1491-1503

Wasmeier C, Hutton JC (1996) Molecular cloning of phogrin, a protein-tyrosine phosphatase homologue localized to insulin secretory granule membranes. J Biol Chem 271:1816118170
Weir GC, Laybutt DR, Kaneto H, Bonner-Weir S, Sharma A (2001) Beta-cell adaptation and decompensation during the progression of diabetes. Diabetes 50(Suppl 1):S154-S159

Wendler F, Page L, Urbe S, Tooze SA (2001) Homotypic fusion of immature secretory granules during maturation requires syntaxin 6. Mol Biol Cell 12:1699-1709 